\title{
THE EQUIVALENCE OF COMPLEX POINTS, PLANES, LINES WITH RESPECT TO REAL MOTIONS AND CERTAIN OTHER GROUPS OF REAL TRANSFORMATIONS*
}

BY

\section{WILLIAM CASPAR GRAUSTEIN}

1. Introduction. This paper has to do with geometry in three-dimensional complex space. The points, planes, lines of this space, the elements of the space in which we shall be interested, are called complex points, planes, lines, or briefly, complex elements. The special problem to be treated in the paper is the equivalence of these complex elements with respect to certain groups of real transformations, and in particular with respect to the group of real motions.

Two like-named complex elements are said to be equivalent with respect to a given group of transformations if there exists a transformation of the group carrying the one into the other. $\dagger$ Two such elements, which are equivalent with respect to the group of real motions in particular, we shall call, for the sake of brevity, real-congruent. Every two real like-named elements are of course real-congruent. But two imaginary $\ddagger$ like-named elements are not, in general, real-congruent. To develop necessary and sufficient conditions for their real-congruence is the main object of this paper.

* Presented to the Society, December 31, 1913, under a different title.

$\dagger$ The groups of real transformations, with respect to which we shall study the equivalence of complex elements, are (1) the group of real motions, (2) the group of real motions and reflections, (3) the group of real transformations of similitude. The equations in point coördinates of the group of $2 \cdot \infty^{7}$ transformations of similitude are

$$
z_{i}^{\prime}=r\left(a_{i} z_{1}+b_{i} z_{2}+c_{i} z_{3}+d_{i}\right), \quad r \neq 0, \quad(i=1,2,3),
$$

where the $a$ 's, $b$ 's, $c$ 's, $d$ 's, and $r$ are real and the determinant of the coefficients of the $z$ 's is orthogonal and has the value +1 . These become the equations, (1) of the group of real motions and reflections, if we set $r= \pm 1$, (2) of the group of real motions alone, if we set $r=+1$.

We shall consider the equivalence of only those elements lying in the finite domain. Ruling out the elements at infinity causes no complications here, since all the groups of transformations considered are subgroups of the affine group.

$\ddagger$ "Real " and "imaginary," as used here and throughout, are mutually exclusive; " complex" covers both. 
2. Classification of points, planes, and lines.* A necessary condition that two complex points be real-congruent is that they be either both real or both imaginary. Accordingly, we divide complex points into two classes, the class of real points and the class of imaginary points.

That two complex planes (or lines) be real-congruent it is evidently necessary, first, that they contain the same number of real points; second, that the distribution of these real points between the finite and infinite domains be the same for both; third, that they should be both minimal or both euclidean (non-minimal). $\dagger$ Accordingly, a first step toward the solution of our problem will be a division into classes of complex planes and lines according to these three methods of distinction.

A complex plane contains either $\infty^{2}$ real points, in which case it is real, or $\infty^{1}$ real points, in which case it is imaginary. The $\infty^{1}$ real points of an imaginary plane are the real points of a real line, the line of intersection of the plane with its conjugate-imaginary plane. If this line is at infinity we shall call the plane low-imaginary; if the line is not at infinity we shall call the plane high-imaginary. (The direction of the normal of a low-imaginary plane is real, that of the normal of a high-imaginary plane is imaginary.) A lowimaginary plane is always euclidean. A high-imaginary plane, however, may be either euclidean or minimal.

Thus we have obtained four classes of planes, which we shall denote by distinctive capital letters, as follows:

\begin{tabular}{lc}
\multicolumn{1}{c}{ Plane } & Designation \\
Real & $R$-plane \\
Low-imaginary & $L$-plane \\
High-imaginary $\ddagger$ minimal & $M$-plane \\
High-imaginary euclidean & $H$-plane
\end{tabular}

A complex straight line contains $\infty^{1}$ real points or lies in $\infty^{1}$ real planes when and only when it is real. An imaginary straight line either $(a)$ contains one real point and lies in one real plane, or $(b)$ contains no real point and lies in no real plane. In case $(a)$ we shall call the line incomplete-imaginary; in case $(b)$, complete-imaginary.

If the real point of an incomplete-imaginary line is at infinity, the line will be termed low-imaginary, otherwise high-imaginary. A low-imaginary line has a real direction and is therefore always euclidean.

* The contents of this section appeared in greater detail in the author's dissertation, Eine reelle Abbildung analytischer komplexer Raumkurven, Bonn, 1913, pp. 4-6. For a discussion of the terminology see footnote (2), p. 4 of this dissertation.

$\dagger$ For the use of "euclidean" in the sense of "non-minimal" see Study, these Tr a $\mathbf{n}$ a c t i o n s, vol. 10 (1909), p. 19.

$\ddagger$ Without causing ambiguity we may omit the adjective " high-imaginary" in the case of $M$-planes. 
Finally we combine the division (of the lines with imaginary directions) into high- and complete-imaginary with the division into euclidean and minimal.

Thus we have in all six classes of lines, to be designated by suggestive capital letters, as follows:

$\begin{array}{lc}\text { Real } & \text { DESIGNATION } \\ \text { Low-imaginary } & R \text {-line } \\ \text { Incomplete-imaginary minimal } & L \text {-line } \\ \text { High-imaginary euclidean } & M \text {-line } \\ \text { Complete-imaginary minimal } & H \text {-line } \\ \text { Complete-imaginary euclidean } & C M \text {-line } \\ & C E \text {-line }\end{array}$

3. Fundamental theorem. We introduce the following notation. The element which is conjugate-imaginary to a given element $T$ shall be denoted by $\bar{T}$, the (shortest) distance from $T$ to $\bar{T}$ by $d(T, \bar{T})$, and the angle between $T$ and $\bar{T}$, measured from $T$ to $\bar{T}$, if it exists, by $\Varangle(T, \bar{T})$. As is well known, $d^{2}(T, \bar{T})$ and, when existent, $\tan ^{2} \Varangle(T, \bar{T})$ are absolute invariants of $T$ and $\bar{T}$ under the group of real motions.*

Fundamental Theorem: The necessary and sufficient conditions that two like-named imaginary elements, $R$ and $S$, be real-congruent are (1) that they belong to the same class, (2) that $d^{2}(R, \bar{R})=d^{2}(S, \bar{S})$, and $(3)$, if $\Varangle(R, \bar{R})$ and $\Varangle(S, \bar{S})$ exist, that $\tan ^{2} \Varangle(R, \bar{R})=\tan ^{2} \Varangle(S, \bar{S}) . \dagger$

The conditions of the theorem are evidently necessary. In proving their sufficiency - the crux of the whole problem-we propose to establish first the theorem analogous to the Fundamental Theorem for each class of complex elements of $(a)$ the real line, and $(b)$ the real plane. These theorems, besides being of interest in themselves, will serve as the basis for the proof of the sufficiency of the Fundamental Theorem.

4. Cases of one and two dimensions. For the one-dimensional geometry of the real line "real-congruence" signifies equivalence with respect to the group of $\infty^{1}$ real translations of the line into itself.

TheoRem 1. Two real points are always real-congruent under one and only one translation.

Theorem 2. If the two imaginary points, $A$ and $B$, are real-congruent, then $d(A, \bar{A})=d(B, \bar{B}) . \ddagger$ Conversely, if $d(A, \bar{A})=d(B, \bar{B}), A$ is carried into $B$ by one and only one real translation.

* An analytical method for setting up these invariants is given in $\$ 7$.

$\dagger$ For the complete-imaginary lines a slight change must be made in condition (2); see Theorems 19, 20. It is also to be noted that either of the conditions (2) or (3) may, in a given case, be no longer restrictive: condition (2) if $R$ and $S$ are members of a class for which $d^{2}(T, \bar{T})=0$ (i. e., if $R$ and $S$ are $M$ - or $H$-planes, or $M$ - or $H$-lines); condition (3) if $R$ and $S$ belong to a class for which $\tan ^{2} \Varangle(T, \bar{T})=0$ (i. e., if $R$ and $S$ are $L$-planes or $L$-lines). Finally the condition (3) drops naturally if $R$ and $S$ are points or minimal elements.

$\ddagger$ The algebraic and not the absolute value of $d$ is meant here, since directed distance is an absolute invariant of translations of a line into itself. Consequently, two conjugateimaginary points are not equivalent with respect to real translations of their line into itself. 
For if $d(A, \bar{A})=d(B, \bar{B})$, the translation carrying the (real) midpoint of $A$ and $\bar{A}$ into the (real) midpoint of $B$ and $\bar{B}$ carries $A$ into $B$. The analytical proof is also simple.

The real plane contains, of the elements in which we are interested, $R$-, $L$-, $M$-, and $H$-lines, besides points. "Real-congruence" signifies here equivalence with respect to the group of $\infty^{3}$ real motions of the plane into itself.

Theorem 3. Two real points, $A$ and $B$, are always real-congruent under $\infty^{1}$ motions (the translation carrying $A$ into $B$, combined with the $\infty^{1}$ rotations about $B$ ).

Theorem 4. Two real lines, $a$ and $b$, are always real-congruent under $2 \cdot \infty^{1}$ motions (two rotations* of $a$ into $b$, combined with $\infty^{1}$ real translations along $b$ ).

Theorem 5. If two imaginary points, $A$ and $B$, are real-congruent,

$$
d^{2}(A, \bar{A})=d^{2}(B, \bar{B}) \text {. }
$$

Conversely, if $d^{2}(A, \bar{A})=d^{2}(B, \bar{B}), A$ is carried into $B$ by one and only one real motion.

For if $d^{2}(A, \bar{A})=d^{2}(B, \bar{B})$, then, of the $2 \cdot \infty^{1}$ real motions carrying the real line $A \bar{A}$ into the real line $B \bar{B}, \infty^{1}$ will have the effect that, in this new position, $d(A, \bar{A})=d(B, \bar{B})$, and of these motions one and only one will carry $A$ into $B$ (cf. Theorem 2 ).

Theorem 6. Two L-lines, $a$ and $b$, are real-congruent only when

and then under $\infty^{1}$ motions.

$$
d^{2}(a, \bar{a})=d^{2}(b, \bar{b}),
$$

For if $d^{2}(a, \bar{a})=d^{2}(b, \bar{b})$, then, of the $2 \cdot \infty^{1}$ motions carrying the (real) line midway between $a$ and $\bar{a}$ into the (real) line midway between $b$ and $\bar{b}$ (cf. Theorem 4), $\infty^{1}$ will carry $a$ into $b$.

Theorem 7. Two M-lines, $a$ and $b$, are real-congruent only when they go through the same circular point at infinity and then under $\infty^{1}$ motions (the translation of the real point of $a$ into the real point of $b$, combined with the $\infty^{1}$ real rotations about the latter point).

Theorem 8. If two $H$-lines, $a$ and $b$, are real-congruent,

$$
\tan \Varangle(a, \bar{a})=\tan \Varangle(b, \bar{b}) \cdot \dagger
$$

Conversely, if $\tan \Varangle(a, \bar{a})=\tan \Varangle(b, \bar{b})$, then there are two and only two real motions carrying a into $b$.

\footnotetext{
* If $a$ and $b$ are parallel, the two rotations are to be replaced by a translation of $a$ into $b$ and a combination of this translation and a rotation through $180^{\circ}$ of $b$ about any of its points.

† Tangent of the angle and not the square of the tangent, since oriented angle is an absolute invariant of motions in a plane. Hence two conjugate-imaginary $H$-lines are not equivalent with respect to the real motions in their plane. This is also true for two conjugateimaginary $M$-lines.
} 
Translate the point of intersection of $a$ and $\bar{a}$ into the point of intersection of $b$ and $\bar{b}$. Then either of the two rotations carrying the real lines bisecting the angles between $a$ and $\bar{a}$ into the corresponding bisectors of the angles between $b$ and $\bar{b}$ will carry $a$ into $b$, provided that $\tan \Varangle(a, \bar{a})=\tan \Varangle(b, \bar{b}) .^{*}$

5. Case of three dimensions. We now proceed to the proof of the Fundamental Theorem for each class of complex elements of three-dimensional space.

Theorem 9. Two real points, $A$ and $B$, are always real-congruent under $\infty^{3}$ motions (the translation carrying $A$ into $B$, combined with the $\infty^{3}$ real rotations about $B$ ).

Theorem 10. Two real planes, $\alpha$ and $\beta$, are always real-congruent under $2 \cdot \infty^{3}$ motions (two rotations $\dagger$ of $\alpha$ into $\beta$ about their line of intersection, combined with $\infty^{3}$ motions leaving $\beta$ invariant).

Theorem 11. Two real lines, $a$ and $b$, are always real-congruent under $2 \cdot \infty^{2}$ motions (a motion carrying $a$ into $b$, combined with the $2 \cdot \infty^{1}$ rotations and $\infty^{1}$ translations leaving $b$ invariant).

Theorem 12. If two imaginary points, $A$ and $B$, are real-congruent,

$$
d^{2}(A, \bar{A})=d^{2}(B, \bar{B}) .
$$

Conversely, if $d^{2}(A, \bar{A})=d^{2}(B, \bar{B}), A$ is carried into $B b y \infty^{1}$ real motions.

The proof is similar to that of Theorem 5 .

ThEOREM 13. Two L-planes, $\alpha$ and $\beta$, are real-congruent only when

and then under $\infty^{3}$ motions.

$$
d^{2}(\alpha, \bar{\alpha})=d^{2}(\beta, \bar{\beta}),
$$

The proof is similar to that of Theorem 6 .

Theorem 14. Any two $M$-planes, $\alpha$ and $\beta$, are real-congruent under $\infty^{2}$ motions.

Of the $2 \cdot \infty^{2}$ real motions carrying the line of intersection of $\alpha$ and $\bar{\alpha}$ into the line of intersection of $\beta$ and $\bar{\beta}$ (cf. Theorem 11), $\infty^{2}$ will carry $\alpha$ into $\beta$.

Theorem 15. If two $H$-planes, $\alpha$ and $\beta$, are real-congruent,

$$
\tan ^{2} \Varangle(\alpha, \bar{\alpha})=\tan ^{2} \Varangle(\beta, \bar{\beta}) .
$$

* An analytical proof follows. Translate the real point of $a$ into the real point of $b$, and then take this point as origin of coördinates. The equations of $a$ and $b$ are respectively

$$
\mu x-i y=0 \quad \text { and } \quad \nu x-i y=0 .
$$

The necessary and sufficient condition that $a$ and $b$ be real-congruent is that the tangent of the angle between $a$ and $b$, namely $i(\mu-\nu) /(1-\mu \nu)$, be real; this results in

$$
(\mu+\bar{\mu}) /(1+\mu \bar{\mu})=(\nu+\bar{\nu}) /(1+\nu \bar{\nu}),
$$

where $\bar{\mu}, \bar{\nu}$ are conjugate-complex to $\mu, \nu$ respectively. But $\tan \chi(a, a)=i(\mu+\bar{\mu}) /(1+\mu \bar{\mu})$, and $\tan \Varangle(b, \bar{b})=i(\nu+\nu) /(1+\nu \bar{\nu})$.

$\dagger$ If the planes are parallel the two rotations are to be replaced by a translation of the first into the second and a combination of this translation and a rotation through $180^{\circ}$ about any real line in the second. 
Conversely, if $\tan ^{2} \Varangle(\alpha, \bar{\alpha})=\tan ^{2} \Varangle(\beta, \bar{\beta})$, then $\alpha$ is carried over into $\beta$ by $2 \cdot \infty^{1}$ real motions.

For if $\tan ^{2} \Varangle(\alpha, \bar{\alpha})=\tan ^{2} \Varangle(\beta, \bar{\beta})$, we may carry the real line of $\alpha$ into the real line of $\beta$ so that in this position $\tan \Varangle(\alpha, \bar{\alpha})=\tan \Varangle(\beta, \bar{\beta})$. Proceed then as in Theorem 8. Finally, any (real) translation in the direction of the real line of $\beta$ will leave $\alpha$ and $\beta$ coincident.

Theorem 16. Two L-lines, $a$ and $b$, are real-congruent only when

$$
d^{2}(a, \bar{a})=d^{2}(b, \bar{b}),
$$

and then under $2 \cdot \infty^{1}$ motions.

Rotate the plane of $a$ and $\bar{a}$ into the plane of $b$ and $\bar{b}$ about their line of intersection in either of the two possible ways. ${ }^{*}$ Then apply Theorem 6 .

Theorem 17. Two M-lines, $a$ and $b$, are always real-congruent under $\infty^{1}$ motions.

Carry the plane of $a$ and $\bar{a}$ into the plane of $b$ and $\bar{b}$ so that $a$ and $b$ will go through the same circular point at infinity. Then apply Theorem 7.

Theorem 18. If two H-lines, $a$ and $b$, are real-congruent,

$$
\tan ^{2} \Varangle(a, \bar{a})=\tan ^{2} \Varangle(b, \bar{b}) .
$$

Conversely, if $\tan ^{2} \Varangle(a, \bar{a})=\tan ^{2} \Varangle(b, \bar{b}), a$ is carried into $b$ by two and only two real motions.

For if $\tan ^{2} \Varangle(a, \bar{a})=\tan ^{2} \Varangle(b, \bar{b})$, we may carry the plane of $a$ and $\bar{a}$ into the plane of $b$ and $\bar{b}$ so that in this position $\tan \Varangle(a, \bar{a})=\tan \Varangle(b, \bar{b})$. Then apply Theorem 8 .

In order to treat the case of $C E$-lines, we associate with any given $C E$-line $a$ a certain $N$-plane $N_{a}$ and a certain $H$-line $H_{a} . \quad N_{a}$ is, namely, the only plane through $a$ having a normal with a real direction; $H_{a}$ is the projection of $a$ upon the real plane midway between $N_{a}$ and $\bar{N}_{a} . H_{a}$ is parallel to $a$, and hence $\tan \Varangle(a, \bar{a})=\tan \Varangle\left(H_{a}, \bar{H}_{a}\right)$. The common perpendicular to $a$ and $\bar{a}$ is the real normal to the real plane of $H_{a}$ in the real point of $H_{a}$; thus it is normal to both $N_{a}$ and $\bar{N}_{a}$ and hence $d(a, \bar{a})=d\left(N_{a}, \bar{N}_{a}\right)$. This distance, as given by the formula $\dagger$ below, is double valued. In order to obtain a singled-valued, i. e., a directed distance, we must assign a determination to a certain radical. This radical occurs also in the expressions for the direction cosines of the common perpendicular to $a$ and $\bar{a}$, We orient this perpendicular $\ddagger$ and thereby direct the distance $d(a, \bar{a})$. It is this

* See preceding footnote.

$\dagger$ Cf. $J$ in the list in $\$ 7$.

$\ddagger$ This we do as follows. If the direction cosines of $a$ are proportional to $p_{1}: p_{2}: p_{3}$, those of $\bar{a}$ are proportional to $p_{1}: \bar{p}_{2}: \bar{p}_{3}$ and those of the common perpendicular to

$$
i\left(p_{2} \bar{p}_{3}-p_{3} \bar{p}_{2}\right): i\left(p_{3} p_{1}-p_{1} \bar{p}_{3}\right): i\left(p_{1} \bar{p}_{2}-p_{2} \bar{p}_{1}\right) \text {. }
$$

We orient this perpendicular by choosing as its direction cosines these quantities each divided by the positive square root of the sum of their squares. 
directed distance that we shall understand henceforth by $d$, when $d$ is applied to $C E$-lines.

Theorem 19. Two CE-lines, $a$ and $b$, are real-congruent only when

$$
d(a, \bar{a})=d(b, \bar{b}) \quad \text { and } \quad \tan ^{2} \Varangle(a, \bar{a})=\tan ^{2} \Varangle(b, \bar{b}),
$$

and then under two and only two motions.

From the preceding considerations it is evident that a real motion carrying $a$ into $b$ must carry $H_{a}$ into $H_{b}$. But there are two and only two real motions carrying $H_{a}$ into $H_{b}$, if $\tan ^{2} \Varangle(a, \bar{a})=\tan ^{2} \Varangle(b, \bar{b})$; for then

$$
\tan ^{2} \Varangle\left(H_{a}, \bar{H}_{a}\right)=\tan ^{2} \Varangle\left(H_{b}, \bar{H}_{b}\right),
$$

and we may apply Theorem 18. Moreover these motions carry the directed common perpendicular to $a$ and $\bar{a}$ into the directed common perpendicular to $b$ and $\bar{b}$. Now if $d(a, \bar{a})=d(b, \bar{b})$, then $d\left(N_{a}, \overline{N_{a}}\right)=d\left(N_{b}, \overline{N_{b}}\right)$, and hence either of the motions carries $N_{a}$ into $N_{b}$ and therefore $a$ into $b$.

The case of the $C M$-lines may be treated similarly; or in this treatment of $C E$-lines, replace $C E$ by $C M, H$ by $M$, use Theorem 17 instead of Theorem 18 in the proof and omit the statements involving angle. We give merely the theorem.

Theorem 20. Two CM-lines, $a$ and $b$, are real-congruent only when

and then under $\infty^{1}$ motions.

$$
d(a, \bar{a})=d(b, \bar{b}),
$$

6. Extension to transformations of symmetry and similitude. We note that, whenever directed distance or angle is an absolute invariant with respect to the group of real motions, it is a relative invariant with -1 as the factor of reproduction with respect to the corresponding set of real reflections, and that, whereas any motion in a real plane leaves each of the minimal directions in this plane invariant, the corresponding reflection interchanges the two minimal directions. If we make changes in accordance with these facts in Theorems 1-20, we obtain necessary and sufficient conditions for equivalence with respect to the set of real reflections.

It is now evident that, if we combine the real motions and reflections into a single group, we may obtain valid theorems for equivalence with respect to this group by replacing, in Theorems 1-20, every equality linear in $d$ or in the tangent of an angle by the corresponding quadratic equality and by omitting the condition of Theorem 7 . Then the number of transformations of the mixed group carrying the first element into the second is, in the case of the altered Theorems $2,7,8,19,20$, the same as the number of real motions effecting the result, but in the case of the other theorems, double* this number.

\footnotetext{
* That is, if $k \cdot \infty^{a}$ is the number of motions, the number of motions and reflections is $2 k \cdot \infty^{a}$.
} 
Finally we recall that, with respect to the group of real transformations of similitude, $d^{2}$ is but a relative invariant. (The square of the tangent of an angle remains an absolute invariant.) Accordingly, to obtain necessary and sufficient conditions for equivalence under this group, we omit in Theorems 1-20 all conditions involving distance (besides, of course, the condition of Theorem 7) and replace $\tan \Varangle(a, \bar{a})=\tan \Varangle(b, \bar{b})$ in Theorem 8 by

$$
\tan ^{2} \Varangle(a, \bar{a})=\tan ^{2} \Varangle(b, \bar{b}) .
$$

The number of real transformations of similitude carrying the first element into the second is in every case $\infty^{1}$ times* the number of real motions and reflections effecting the result.

7. A complete system of rational invariants. During the remainder of the paper we shall restrict our considerations to three-dimensional space. Analogous work for the real line and the real plane is so simple as hardly to merit explicit treatment here and at the same time is, for the most part, deducible from the discussion of the three dimensional case.

The conditions given in Theorems 9-20 for the real-congruence of complex elements are based on absolute invariants of these elements under the group of real motions. We now give the analytical expression for each of these invariants (also the interval of values it takes on for the class for which it is given). $†$

Imaginary points $Z \equiv(\overline{z-\bar{z}} \mid \overline{z-\bar{z}})=d^{2}(z, \bar{z}), \quad-\infty<Z<0$,

L-planes

$$
\begin{aligned}
& L_{p} \equiv \frac{w_{0}^{2}}{(w \mid w)}-\frac{2 w_{0} \bar{w}_{0}}{(w \mid \bar{w})}+\frac{\bar{w}_{0}^{2}}{(\bar{w} \mid \bar{w})}=d^{2}(w, \bar{w}) \\
& -\infty<L_{p}<0 \text {, }
\end{aligned}
$$

$H$-planes

$$
I_{p} \equiv \frac{(w \bar{w} \mid w \bar{w})}{(w \mid \bar{w})^{2}}=\tan ^{2} \Varangle(w, \bar{w}),
$$$$
-1<I_{p}<0 \text {, }
$$

$L$-lines

$$
L_{l} \equiv \frac{(q \mid q)}{(p \mid p)}-2 \frac{(q \mid \bar{q})}{(p \mid \bar{p})}+\frac{(\bar{q} \mid \bar{q})}{(\bar{p} \mid \bar{p})}=d^{2}(p: q, \bar{p}: \bar{q})
$$

$H$-lines

$$
I_{l} \equiv \frac{(p \bar{p} \mid p \bar{p})}{(p \mid \bar{p})^{2}}=\tan ^{2} \Varangle(p: q, \bar{p}: q),
$$

$$
-\infty<L_{l}<0,
$$$$
-1<I_{l}<0 \text {, }
$$

* That is, if $k \cdot \cdot^{a}$ is the number of motions and reflections, the number of transformations of similitude is $k \cdot \infty^{a+1}$.

$\dagger$ Here and later we use $z_{1}, z_{2}, z_{3}$ as the coördinates of a point $z$, and $w_{0}, w_{1}, w_{2}, w_{3}$ as the homogeneous coördinates of a plane $w$; also $p_{1}, p_{2}, p_{8} ; q_{1}, q_{2}, q_{3}$ as the plueckerian coördinates of a line $p: q$. Further, if $r:\left(r_{1}, r_{2}, r_{3}\right), s:\left(s_{1}, s_{2}, s_{3}\right)$, etc., are any triples of numbers, we denote the triple $\left(r_{2} s_{3}-r_{3} s_{2}, r_{3} s_{1}-r_{1} s_{3}, r_{1} s_{2}-r_{2} s_{1}\right)$ by $\hat{r}$, and introduce the symbols $(r \mid s),(r s \mid r s),(r s t)$, where

$$
\begin{aligned}
& (r \mid s) \equiv r_{1} s_{1}+r_{2} s_{2}+r_{3} s_{3}, \\
& (r s \mid r s) \equiv(\hat{r} \mid \hat{r s}) \text {, } \\
& (r s t) \equiv\left|\begin{array}{lll}
r_{1} & r_{2} & r_{3} \\
s_{1} & s_{2} & s_{3} \\
t_{1} & t_{2} & t_{3}
\end{array}\right| .
\end{aligned}
$$


$C M$-lines

$$
J \equiv i \frac{(p \mid \bar{q})+(\bar{p} \mid q)}{\sqrt{-(p \bar{p} \mid p \bar{p})} *}=d(p: q, \bar{p}: \bar{q}), \quad 0<J^{2}<\infty,
$$

$C E$-lines

$I$ and $J$

$$
-1<I_{l}<0,0<J^{2}<\infty \text {. }
$$

To derive these expressions one has but to evaluate $d, d^{2}$ and $\tan ^{2} \Varangle$ in the several cases. But since the expressions are absolute invariants under the group of real motions, they should be obtainable from the equations of this group. We proceed to derive $I_{l}, J$, and $L_{l}$ in this way; the derivation of the others is similar.

The equations of the group of real motions in line coördinates are

$$
\begin{gathered}
\rho\left(p^{\prime} \mid \omega\right) \overline{\bar{\omega}}(a \mid \omega) p_{1}+(b \mid \omega) p_{2}+(c \mid \omega) p_{3}, \quad(\rho, \text { complex, } \neq 0), \\
\rho\left(q^{\prime} \mid \omega\right) \bar{\equiv}(d a \omega) p_{1}+(d b \omega) p_{2}+(d c \omega) p_{3} \\
+(b c \omega) q_{1}+(c a \omega) q_{2}+(a b \omega) q_{3} \cdot \dagger
\end{gathered}
$$

From the first identity we have

$$
\begin{aligned}
& \rho^{2}\left(p^{\prime} \mid p^{\prime}\right) \equiv(p \mid p), \\
& \rho \bar{\rho}\left(p^{\prime} \mid \bar{p}^{\prime}\right) \equiv(p \mid \bar{p}) .
\end{aligned}
$$

We may combine (2) and (3) into

$$
(\rho \bar{\rho})^{2}\left(p^{\prime} \bar{p}^{\prime} \mid p^{\prime} \bar{p}^{\prime}\right) \equiv(p \bar{p} \mid p \bar{p}) .
$$

From (3) and (4) we then have

$$
I_{l} \equiv \frac{\left(p^{\prime} \bar{p}^{\prime} \mid p^{\prime} \bar{p}^{\prime}\right)}{\left(p^{\prime} \mid \bar{p}^{\prime}\right)^{2}} \equiv \frac{(p \bar{p} \mid p \bar{p})}{(p \mid \bar{p})^{2}}
$$

Using both identities of (1) we obtain

$$
\rho \bar{\rho}\left[\left(p^{\prime} \mid \bar{q}^{\prime}\right)+\left(\bar{p}^{\prime} \mid q^{\prime}\right)\right] \equiv(p \mid \bar{q})+(\bar{p} \mid q) .
$$

From (4) and (5) we may now show that $J$ is an absolute invariant; but in doing this we must rule out the lines with real directions, since for them $(p \bar{p} \mid p \bar{p})=0$. Thus $J$ is defined only for lines with imaginary directions and has the further disadvantage of being irrational. Now dividing (5) by (3) yields the absolute invariant

$$
K \equiv \frac{(p \mid q)+(p \mid \bar{q})}{(p \mid \bar{p})},
$$

which has neither of these drawbacks. We use it from now on in preference to $J$.

* We take the positive value of this square root.

$\dagger$ Each of these identities in $\omega$, i. e., in $\omega_{1}, \omega_{2}, \omega_{3}$, represents three equations, namely those obtained by equating the coefficients of the two members of the identity for $i=1,2,3$. 
To deduce $L_{l}$ we must first restrict $p: q$ to be a line with a real direction. This done, the left-hand members of the two identities,

$$
\begin{aligned}
& p_{1}(d a \omega)+p_{2}(d b \omega)+p_{3}(d c \omega) \equiv \overline{\bar{\omega}} \rho\left(q^{\prime} \mid \omega\right)-(b c \omega) q_{1}-(c a \omega) q_{2}-(a b \omega) q_{3}, \\
& \bar{p}_{1}(d a \omega)+\bar{p}_{2}(d b \omega)+\bar{p}_{3}(d c \omega) \overline{\bar{\omega}} \bar{\rho}\left(\bar{q}^{\prime} \mid \omega\right)-(b c \omega) \bar{q}_{1}-(c a \omega) \bar{q}_{2}-(a b \omega) \bar{q}_{3},
\end{aligned}
$$

are proportional. Eliminating the left hand members and substituting for $\rho$ and $\bar{\rho}$ their values from (2), we have

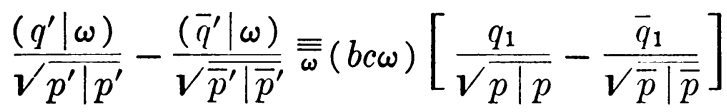

$$
\begin{aligned}
& +(c a \omega)\left[\frac{q_{2}}{\sqrt{p} \mid \bar{p}}-\frac{\bar{q}_{2}}{\sqrt{\bar{p} \mid \bar{p}}}\right]+(a b \omega)\left[\frac{q_{3}}{\sqrt{p \mid p}}-\frac{\bar{q}_{3}}{\sqrt{\bar{p} \mid \bar{p}}}\right],
\end{aligned}
$$

holding for proper determinations of the radicals. From this identity we obtain $L_{l}$ as an absolute invariant.

In the deduction of $I_{l}$ and $K, p: q$ was unrestricted. Hence $I_{l}$ and $K$ are defined, i. e., have meaning as invariants, for every class of lines. Similar statements hold for $Z$ and $I_{p}$. On the other hand $L_{l}$ is defined (as an invariant) only when $p: q$ is a line of real direction; similarly for $L_{p}$.

Now in the list given each invariant was given only for a particular class (or classes) and the (open) interval of the values it takes on just for this class (or classes) was added. For the other classes for which it is defined, the invariant has the constant value (or values) represented by the finite end point (or points) of this interval. Thus $I_{p}$ is listed only for the class of $H$ planes, for which $-1<I_{p}<0$; but for all $M$-planes $I_{p}=-1$ and for all $R$ - and $L$-planes $I_{p}=0$.* $^{*}$

On the basis of Theorems 9-20 we conclude that the invariant (or invariants) given for any class of complex elements forms (or form) a complete system of absolute rational invariants of the general element $\dagger$ of this class under the group of real motions. $[J$ (irrational) is supposed to have been replaced

* For the values of any invariant for each of the classes of elements for which it is defined, see the tables in $\S 8$.

† Or better, from the point of view of analytical invariants, of a pair of conjugate-imaginary elements of the class. For there exists no analytical invariant of a single complex element, i. e., no expression, rational in the coördinates of a single complex element, which is an invariant. Suppose, for instance, that $f\left(z_{1}, z_{2}, z_{3}\right)$ were an invariant of the complex point $z$. Since $z$ and $\bar{z}$ are real-congruent, $f\left(z_{1}, z_{2}, z_{3}\right) \equiv f\left(\bar{z}_{1}, \bar{z}_{2}, \bar{z}_{3}\right)$, whence follows that $f$ must be constant. Similar reasoning shows that an invariant of a pair of conjugate-imaginary elements is a symmetrical function of the two sets of coördinates; consequently we may choose such invariants so that they will be everywhere real-valued.

These considerations bring into prominence a fact which was already evident, namely that, in solving the problem of the real-congruence of two complex elements, we have really solved a slightly more general problem, namely that of the real-congruence of two ordered pairs of conjugate-complex elements. 
by $K$.] For if there existed an (absolute) rational invariant of a complex element not expressible in terms of the listed invariant (or invariants) of the class to which this element belongs (and, of course, not becoming trivial for the class, i. e., not taking on a constant value for all its members), then two elements of the class would not be real-congruent unless they had the same value for this new invariant; this contradicts the theorem on real-congruence for the class in question.

8. Summary and tabulation of results. We shall now subdivide each class of elements into subclasses, where a subclass is the totality of all elements of a class for which the (absolute) invariants defined for the class have the same value. Since the invariants defined for any class not listed in the table in $\S 7$ have constant values for all elements of the class, such a class contains but one subclass, namely itself. Of the classes given in the table, that of the $C E$-lines contains a double infinity of subclasses and each of the remaining ones a single infinity of subclasses.

We may now restate the Fundamental Theorem $(\S 3)$ in better form.

Fundamental Theorem. A necessary and sufficient condition that two like-named complex elements be real-congruent is that they belong to the same subclass.

The following tables give a summary of our results in the form of a division, first of the complex elements into classes and then of the classes into subclasses; the last column gives the number of motions under which two elements of one of the subclasses of the class in question are real-congruent.

\begin{tabular}{cccccc} 
Class & $\begin{array}{c}\text { No. of points } \\
\text { in class }\end{array}$ & $\begin{array}{c}\text { Value } \\
\text { for } \boldsymbol{Z}\end{array}$ & $\begin{array}{c}\text { No. of } \\
\text { subclasses }\end{array}$ & $\begin{array}{c}\text { No. in one } \\
\text { subclass }\end{array}$ & $\begin{array}{c}\text { No. of } \\
\text { motions }\end{array}$ \\
\hline $\begin{array}{c}\text { Real } \\
\text { Imaginary }\end{array}$ & $\infty^{3}$ & 0 & One & $\infty^{3}$ & $\infty^{3}$ \\
\hline
\end{tabular}

\begin{tabular}{|c|c|c|c|c|c|c|c|}
\hline \multirow[b]{2}{*}{ Class } & \multicolumn{6}{|c|}{ Planes } & \multirow[b]{2}{*}{$\begin{array}{r}\text { No. of } \\
\text { motions }\end{array}$} \\
\hline & $\begin{array}{l}\text { No. of planes } \\
\text { in class }\end{array}$ & & $\begin{array}{l}\text { Value } \\
\text { for } L_{p}\end{array}$ & $\begin{array}{l}\text { Value } \\
\text { for } I_{p}\end{array}$ & $\begin{array}{c}\text { No. of } \\
\text { subclasses }\end{array}$ & $\begin{array}{c}\text { No. in one } \\
\text { subclass }\end{array}$ & \\
\hline$R$ & $\infty^{3}$ & & 0 & 0 & One & $\infty^{3}$ & $2 \cdot \infty^{3}$ \\
\hline$L$ & $\infty^{4}$ & $-\infty$ & $<L_{p}<0$ & 0 & $\infty^{1}$ & $\infty^{3}$ & $\infty^{3}$ \\
\hline$M$ & $\infty^{4}$ & & & -1 & One & $\infty^{4}$ & $\infty^{2}$ \\
\hline$H$ & $\infty^{6}$ & & - & $<I_{p}<0$ & $\infty^{1}$ & $\infty^{5}$ & $2 \cdot \infty^{1}$ \\
\hline
\end{tabular}

\section{LINES}

\begin{tabular}{cccccccc} 
Class & $\begin{array}{c}\text { No. of lines Value } \\
\text { in class }\end{array}$ & $\begin{array}{c}\text { Value } \\
\text { for } L_{l}\end{array}$ for $I_{l}$ & $\begin{array}{c}\text { Value } \\
\text { for } K\end{array}$ & $\begin{array}{c}\text { No. of } \\
\text { subclasses }\end{array}$ & $\begin{array}{c}\text { No. in one } \\
\text { subclass }\end{array}$ & $\begin{array}{c}\text { No. of } \\
\text { motions }\end{array}$ \\
\hline$R$ & $\infty^{4}$ & 0 & 0 & 0 & One & $\infty^{4}$ & $2 \cdot \infty^{2}$ \\
$L$ & $\infty^{6}$ & $\neq 0$ & 0 & 0 & $\infty^{1}$ & $\infty^{5}$ & $2 \cdot \infty^{1}$ \\
$M$ & $\infty^{5}$ & & -1 & 0 & 0 ne & $\infty^{5}$ & $\infty^{1}$ \\
$H$ & $\infty^{7}$ & $-1<I_{l}<0$ & 0 & $\infty^{1}$ & $\infty^{6}$ & 2 \\
$C M$ & $\infty^{6}$ & $-1<I_{l}<0$ & $\neq 0$ & $2 \cdot \infty^{1}$ & $\infty^{5}$ & $\infty^{1}$ \\
$C E$ & $\infty^{8}$ & $-1<$ & $2 \cdot \infty^{2}$ & $\infty^{6}$ & 2 \\
\hline
\end{tabular}


Inspection of the last two columns in each table shows us that the number of dimensions of the manifold of complex elements forming a subclass plus the number of dimensions of the manifold of real motions, under which two elements of the subclass are real-congruent, is always equal to six, the number of dimensions of the group of real motions.

Similar tables (from which we may draw similar conclusions) may be constructed to exhibit the results of $\S 6$ for the equivalence of complex elements with respect to (1) the group of real motions and reflections, (2) the group of real transformations of similitude. In this connection we note that, of the absolute invariants under the group of real motions, only $I_{p}$ and $I_{l}$ are absolute invariants under the group of real transformations of similitude. For the others we have

$$
Z^{\prime}=r^{2} Z, \quad L_{p}^{\prime}=r^{2} L_{p}, \quad L_{l}^{\prime}=r^{-2} L_{l}, \quad K^{\prime}=r K{ }^{*}
$$

For the group of real motions and reflections, $r= \pm 1$; hence all but $K$ are absolute invariants under this group and $K$ is replaced by $K^{2}$.

HARVARD UNIVERSITY,

Cambridge, Massachusetts,

March, 1914.

* For the meaning of $r$, see the second footnote of the paper. 